\title{
Degradation of Micropollutants Using Advanced Oxidation Processes - From Process Fundamentals to Municipal and Industrial Wastewater Applications
}

\author{
Mohamed Gamal El-Din, Ph.D., P.Eng., FCSCE \\ Department of Civil and Environmental Engineering \\ University of Alberta
}

\begin{abstract}
In recent years, micropollutants such as pharmaceuticals, personal care products, and pesticides have been increasingly detected in the aquatic environment. Micropollutants derived from hormonally active substances have been reported to cause feminization of fish due to their persistence and bioaccumulation potentials. In addition, the effects of long-term exposure to these contaminants remain largely unknown with respect to ecological and human health. There are raised concerns around the world about the existence of micropollutants such as diclofenac, ibuprofen, and estrone at high concentrations. Moreover, antibacterials such as sulfonamide, enrofloxacin and chlorotetracycline deserve a special attention for their removal as their continuous discharge into drinking water sources may increase the risk of development of resistant bacteria strains, affecting the human health. Similarly, emerging persistent contaminants such as perfluorooctane sulfonate (PFOS) have become a global concern because of their toxicity, bioaccumulative properties, and their presence in natural waster as well as in municipal wastewater effluents. Advanced oxidation processes (AOPs) have been widely demonstrated to be reliable for wastewater treatment since they have a high capacity to oxidize nearly all organic pollutants. This capacity derives from the generation of hydroxyl radicals $(\bullet \mathrm{OH})$, sulfate radicals (SO4-•), and other highly reactive oxygen species. Studies have shown that ozone-based AOPs are efficient for the removal of a number of micropollutants in water and wastewater. Persulfatebased AOPs have also received increasing interests due to the high redox potential of the regenerated SO4-• compared with that of $\bullet \mathrm{OH}$ radicals. Although the literature has reported the efficiency of catalytic AOPs to degrade a wide range of micropollutants, a disadvantage of AOPs is the generation of byproducts that may be more toxic than parent compounds. Even though several studies have generated important knowledge on catalytic oxidation, the mechanisms of removal are not well understood possibly due to the different properties of the adsorbents/catalysts and the properties of the target pollutants. This presentation will give an overview of the main advantages and drawbacks of some advanced oxidation processes for micropollutant removals. Detailed explanation of important advancements in this area and future directions will also be provided.
\end{abstract}

